UDEM-GPP-TH-95031

\title{
Baby Skyrmion Strings
}

\author{
by T. Gisiger and M. B. Paranjape \\ Groupe de Physique des Particules, Laboratoire de physique nucléaire, Université de Montréal \\ C.P. 6128, succ. centre-ville, Montréal, Québec, Canada, H3C 3J7
}

\begin{abstract}
We provide analytical and numerical evidence of the existence of classically stable, string-like configurations in a $2+1$ dimensional analog of the Skyrme model. The model contains a conserved topological charge usually called the baryon number. Our strings are non-topological solitons which have a constant baryon number per unit length. The energy per length containing one baryon is, however, less than the energy of an isolated baryon (radially symmetric "baby Skyrmion") in a region of the parameter space, which suggests a degree of stability for our configurations. In a limiting case, our configuration saturates a Bogomolnyi-type bound and is degenerate in energy per baryon with the baby Skyrmion. In another limiting case, the energies are still degenerate but do not saturate the corresponding Bogomolnyi-type bound. Nonetheless, we expect the string to be stable here. Both limiting cases are solvable analytically.
\end{abstract}

The Skyrme model ${ }^{1}$ is a non-linear sigma model containing topological solitons which describe the low-energy dynamics of mesons and baryons. Its $2+1$ dimensional analog was studied in various contexts. Here the fields take values in the two-sphere $S^{2}$, hence the dynamical variables correspond to maps from $R^{2}$ to $S^{2}$. Imposing that all configurations go to a constant at spatial infinity effectively compactifies the spatial $R^{2}$ also into an $S^{2}$, 
and hence all maps are characterized by a topological charge corresponding to the winding number of maps from $S^{2}$ to $S^{2}$. With the addition of the Hopf term to the action, it was shown that the solitons have fractional spin and statistics ${ }^{2}$. The stability and dynamics of the solitons of the model, called baby Skyrmions, have been studied ${ }^{3}$ and it was shown that stable localised solitons exist with the incorporation of a Skyrme term and a mass term. For a study of many solutions of the $C P^{1}$ model in $2+1$ dimensions, see reference [4]. Soliton-soliton scattering has been studied in reference [5] which gives a simpler analog of the corresponding phenomenon in the usual Skyrme model ${ }^{6}$. In a condensed matter context it was shown that $2+1$ dimension Skyrmions are also relevant in quantum Hall systems and anyonic superconductors ${ }^{7}$.

The energy functional is given by the usual kinetic term of the $O(3)$ non-linear sigma model $^{8}$, a four derivative term which is analogous to the Skyrme term ${ }^{9,3}$, and a mass term (or interaction with an external magnetic field) which actually breaks the symmetry and picks out a vacuum ${ }^{10}$,

$E=\frac{1}{2} \int d^{2} \vec{x}\left[\partial_{i} \vec{\phi}(x) \cdot \partial_{i} \vec{\phi}(\vec{x})+\left(\partial_{1} \vec{\phi}(\vec{x}) \times \partial_{2} \vec{\phi}(\vec{x})\right)^{2}+\mu^{2}(\vec{n}-\vec{\phi}(\vec{x}))^{2}\right]$.

Here $\phi^{a}, a=1,2,3$ are the components of a unit vector $\vec{\phi}, \vec{\phi}(\vec{x}) \cdot \vec{\phi}(\vec{x})=1$, and $\vec{n}$ is a constant unit vector taken for convenience to be $(0,0,1)$. The kinetic term along with the Skyrme term are not sufficient to stabilize a baby Skyrmion contrary to the usual Skyrme model. The kinetic term in $2+1$ dimensions enjoys (suffers from) conformal invariance and the baby Skyrmion can always reduce its energy by inflating (infinitely). Hence one adds the mass term which limits the size of the baby Skyrmion. The usual Skyrme term of course prohibits the collapse of the soliton.

The configuration giving rise to a baby Skyrmion with topological charge $N$ is (see the first article of reference [2])

$\vec{\phi}(r, \theta)=(\sin f(r) \cos N \theta, \sin f(r) \sin N \theta, \cos f(r))$

where $f(r)$ goes from $\pi$ at the origin to zero at $+\infty$, and where the topological charge, which by analogy, we call the baryon number, is given by 
$B=\frac{1}{4 \pi} \int d^{2} \vec{x} B(\vec{x})=\frac{1}{4 \pi} \int d^{2} \vec{x} \vec{\phi} \cdot \partial_{1} \vec{\phi}(\vec{x}) \times \partial_{2} \vec{\phi}(\vec{x})$

This gives (for $N=1$ ) the ordinary, non-linear differential equation for $f(r)^{3}$ :

$$
\begin{array}{r}
\left(r+\frac{\sin ^{2} f(r)}{r}\right) f^{\prime \prime}(r)+\left(1-\frac{\sin ^{2} f(r)}{r^{2}}+\frac{f^{\prime}(r) \sin f(r) \cos f(r)}{r}\right) f^{\prime}(r) \\
-\frac{\sin f(r) \cos f(r)}{r}-r \mu^{2} \sin f(r)=0 .
\end{array}
$$

This equation can only be integrated numerically, except in some limiting cases. A solution with unit baryon number $B$, has $f(0)=\pi$, descending with a finite slope at the origin and arriving to zero $(\bmod 2 \pi)$ at infinity with an exponential, two-dimentional Yukawalike fall-off governed by the mass term. The solution is singular in the sense that it is not differentiable at the origin, however, the energy density is well defined everywhere.

To construct the string-like configuration, we simply re-interpret the radial coordinate $r$ and the angular coordinate $\theta$ as two Cartesian coordinates: $(r, \theta) \rightarrow(x, y)$. This has the effect of laying the configurations which previously occured along rays, from the origin to $r=+\infty$, in a linear progression along the $y$ axis with the value $(0,0,-1)$ at $x=0$ and the value $(0,0,1)$ (the vacuum) at $x=+\infty$. This way we obtain one baryon per length $2 \pi$ in the $y$ direction for the right half plane. Along the $y$ axis the configuration has the value $(0,0,-1)$ which is not in the vacuum direction. We must extend our configuration into the left half plane in a way that we also reach the vacuum at $x=-\infty$. The symmetric fashion of achieving this is to glue on to the line at $x=0$ a configuration which corresponds to creating the same string-like configuration as before, however, after having performed a rotation by $\pi$. This rotation has the effect of reversing the directions of $x$ and $y$. Indeed the configuration, where we have rescaled $y$,

$\vec{\phi}(x, y)=\left(\sin f(x) \cos \frac{\pi y \operatorname{sign}(x)}{L}, \sin f(x) \sin \frac{\pi y \operatorname{sign}(x)}{L}, \cos f(x)\right)$

with $f(0)=\pi, f( \pm \infty)=0 \bmod 2 \pi$, has unit baryon number per length $L$ and has a baryon number density which is independent of $y$ :

$B(x, y)=\frac{\pi \operatorname{sign}(x)}{L} \sin f(x) f^{\prime}(x)$. 
The function $f(x)$ satisfies the differential equation

$$
\left(1+\left(\frac{\pi}{L}\right)^{2} \sin ^{2} f(x)\right) f^{\prime \prime}(x)+\left(\frac{\pi}{L}\right)^{2} \frac{\sin 2 f(x)}{2}\left(f^{\prime}(x)^{2}-1\right)-\mu^{2} \sin f(x)=0 .
$$

The solution is found in the right half plane on imposing the boundary condition $f(0)=\pi$, $f(+\infty)=0$. The continuation to the left half plane is done by either reflecting $f(-|x|)=$ $f(|x|)$ so that $f(-\infty)=0$ or continuing smoothly the solution which then interpolates from $f(0)=\pi$ to $f(-\infty)=2 \pi$. Either interpolation gives the same energy and baryon number density. Equation (7) can actually be integrated analytically giving rise to the quadrature:

$\int \sqrt{\frac{1+\pi^{2} / L^{2} \sin ^{2} f}{2 \mu^{2}(1-\cos f)+\pi^{2} / L^{2} \sin ^{2} f}} d f=-x$.

Equation (8) is not terribly useful to obtain $f(x)$, since we still must invert the function defined in terms of the integral on the left hand side, however, we may use it to obtain the energy per baryon as the following integral:

$$
E_{\text {string }}=8 L \int_{0}^{1} d y\left(1+\frac{4 \pi^{2}}{L^{2}}\left(1-y^{2}\right) y^{2}\right)^{1 / 2}\left(\mu^{2}+\frac{\pi^{2}}{L^{2}} y^{2}\right)^{1 / 2}
$$

This allows for a numerical calculation of the energy per baryon without recourse to numerical resolution of any differential equation. Subsequently we minimize with respect to $L$ to find the actual minimum energy string configuration. We find $E_{\text {string }}=1.55356 \times 4 \pi$ with $L_{\text {string }}=3.4542$ for the value of the mass parameter $\mu^{2}=0.1$ as chosen in the second article of reference [3] whereas for the baby Skyrmion $E_{\text {Skyrmion }}=1.564 \times 4 \pi$. Comparing $E_{\text {string }}$ to $E_{\text {Skyrmion }}$ (obtained numerically using the shooting method) for general $\mu^{2}$ we find the following curve for $E_{\text {string }}-E_{\text {Skyrmion }}$ (see Figure 1). This means that the string will be stable against disintegration into individual baryons in the region $0<\mu<1$. The upper limit, $\mu=1$, is obtained numerically with an error of less than $0.002 \%$, but we lack an analytical understanding of this fact. The string with baryon number $N$ is obtained from (5) by the straightforward substitution $y \rightarrow N y$. It is evident from the structure of the ansatz that a string with baryon number $N$ has length $N L_{\text {string }}$ and energy $N E_{\text {string }}$. 
Taking the limit $\mu \rightarrow 0$ while rescaling the coordinates appropriately, removes the Skyrme and mass terms, and we obtain the conformally invariant $O(3)$ non-linear sigma model with energy

$E=\frac{1}{2} \int d^{2} \vec{x} \partial_{i} \vec{\phi}(\vec{x}) \cdot \partial_{i} \vec{\phi}(\vec{x})$

As is well known, this can be written in a form making the Bogomolnyi bound ${ }^{11}$ apparent

$E=\frac{1}{2} \int d^{2}\left[\vec{x}\left(\partial_{1} \vec{\phi}(\vec{x}) \pm \vec{\phi} \times \partial_{2} \vec{\phi}(\vec{x})\right)^{2} \pm 2 B(\vec{x})\right]$

Hence $E \geq 4 \pi|B|$, the Bogomolnyi bound is saturated when

$\partial_{1} \vec{\phi}(\vec{x}) \pm \vec{\phi}(\vec{x}) \times \partial_{2} \vec{\phi}(\vec{x})=0$

depending on the sign of the baryon number. This equation has a solution for the string configuration, the equation of motion being

$f^{\prime}(x) \mp \frac{\pi \operatorname{sign}(x)}{L} \sin f(x)=0$

with solution

$f(x)=2 \arctan \left(\alpha e^{ \pm \pi|x| / L}\right)$

where $\alpha$ is an arbitrary scale parameter. The solution (14) shows that each half string becomes infinitely wide achieving $\pi$ at $x=+\infty$ for the $+\operatorname{sign}$, and at $x=-\infty$ for the sign. Equation (12) also has a baby Skyrmion type solution, satisfying the equation

$f^{\prime}(r)= \pm \frac{\sin f(r)}{r}$

and given by

$f(r)=2 \arctan \left[(\alpha r)^{ \pm 1}\right]$

The $N$ baryon generalization also satisfies the Bogomolnyi bound. Since the string saturates the Bogomolnyi bound per baryon, it is degenerate per baryon with a configuration of an 
isolated $N$ baryon solution that also saturates this bound. Hence the string configuration is classically stable in this limit. To elaborate this further we simply calculate the energy for a configuration $\vec{\phi}=\vec{\phi}_{0}+\vec{\delta} \phi$, where $\vec{\phi}_{0}$ satisfies the equation (13) or (15), using the expression

$$
\begin{aligned}
E(\phi) & =E\left(\phi_{0}\right)+\int d^{2} \vec{x}\left[\partial_{1} \delta \vec{\phi} \pm\left(\vec{\phi}_{0} \times \partial_{2} \delta \vec{\phi}+\delta \vec{\phi} \times \partial_{2} \vec{\phi}_{0}+\delta \vec{\phi} \times \partial_{2} \delta \vec{\phi}\right)\right]^{2} \\
& =E\left(\phi_{0}\right)+\delta E
\end{aligned}
$$

The energy of the fluctuation $\delta E$ is clearly a positive, semi-definite quantity.

The other limit, essentially $\mu \rightarrow+\infty$ also yields an interesting and analytically solvable system. Here, if we scale the coordinates appropriately while taking the limit, we can dispense with the kinetic term, leaving the energy functional

$E=\frac{1}{2} \int d^{2} \vec{x}\left[\left(\partial_{1} \vec{\phi} \times \partial_{2} \vec{\phi}\right)^{2}+\mu^{2}(\vec{n}-\vec{\phi})^{2}\right]$

The equations of motion with the ansatz (2) and (5) are equally well integrable. Indeed for the radially symmetric situation we get

$f^{\prime \prime}(r) \frac{\sin f(r)}{r}+f^{\prime}(r)^{2} \frac{\cos f(r)}{r}-f^{\prime}(r) \frac{\sin f(r)}{r^{2}}-\mu^{2} r=0$

which integrates to

$-\cos f(r)=\frac{\mu^{2} r^{4}}{8}-\mu r^{2}+1$

with the boundary conditions $f(0)=\pi$, and $f(r) \rightarrow 0$ as $r$ becomes large. The interesting feature of this solution is that the field achieves $(0,0,1)$ exactly at a finite radius $R, f(R)=0$. We fix $R$ by minimizing the energy which amounts to the condition that the configuration achieves $(0,0,1)$ smoothly and yields $R=2 / \sqrt{\mu}$. We glue on to the outside for $r>R$ the vacuum. The energy turns out to be

$E_{\text {Skyrmion }}=\frac{4}{3} 4 \pi \mu$.

For the $N$ baryon ansatz (2)), this energy is simply multiplied by $N$. For the ansatz (5), we obtain the following equation of motion 
$f^{\prime \prime}(x)\left(\frac{\pi}{L}\right)^{2} \sin ^{2} f(x)+f^{\prime}(x)^{2}\left(\frac{\pi}{L}\right)^{2} \frac{\sin 2 f(x)}{2}-\mu^{2} \sin f(x)=0$

which also is trivially integrated,

$-\cos f(x)=\left(\frac{\mu L}{\pi}\right)^{2} \frac{x^{2}}{2}-2\left(\frac{\mu L}{\pi}\right) x+1$

exhibiting the same, intriguing behaviour that the soliton cuts off at a finite half-width $W=2 \pi /(\mu L)$. The energy per baryon is also given by

$E_{\text {string }}=\frac{4}{3} 4 \pi \mu$.

Interestingly enough, this does not depend on $L$, the length per baryon, and the energy per baryon of a thin string is equal to that of a wide string. The area per baryon is also independent of the value of $L$

$A_{\text {string }}=2 \times L \times W=\frac{4 \pi}{\mu}$.

Amazingly enough this corresponds exactly to the area occupied by the radially symmetric baby Skyrmion, $A_{\text {Skyrmion }}=\pi R^{2}=4 \pi / \mu$. We have not been able to unearth the reason for this apparent equality of areas, and also for the apparent shape invariance of the string itself. There is perhaps an underlying area preserving diffeomorphism invariance in the model resulting in this "incompressibility".

This limiting case does in fact contain a Bogomolnyi type bound, which is not saturated. Indeed,

$E=\frac{1}{2} \int d^{2} \vec{x}\left[\left(\partial_{1} \vec{\phi} \times \partial_{2} \vec{\phi} \pm \mu(\vec{n}-\vec{\phi})\right)^{2} \pm 2 \mu^{2} B(\vec{x})\right]$

and the extra cross term in the perfect square involving $\vec{n}$ integrates to zero since it is a total divergence. Hence the energy per baryon satisfies

$E \geq 4 \pi \mu|B|$

In any case, since our strings per baryon number are degenerate with an isolated baryon, and we actually do not expect a lower energy configuration in this sector, our configurations seem to be stable against disintegration into single baryons. 
The classical stability analysis for general $\mu$ is not straightforward. For $\mu \in[0,1]$ we have found that the string is energetically stable against disintegration into single baryons. This is not valid for disintegration into configurations with $B=2$. Taking the spherical ansatz for this "baby deuteron", $n=2$ in (2) and solving (numerically) the corresponding differential equation for $f(r)$, yields a configuration that is quite tightly bound,

$E_{\text {deuteron }}=2 E_{\text {Skyrmion }}-\Delta$.

For example with $\mu^{2}=0.1$ we get $\Delta=0.191 \times 4 \pi$, as found in the second article of reference [3]. Hence, if not classically, we expect a string to be able to quantum mechanically tunnel into a configuration of isolated lumps with $B=2$. Calculation of the tunelling rate requires a detailed understanding of the potential on the space of configurations, which for the moment is lacking. In the two limiting cases studied above, the baby deuteron is degenerate with the string (taken in lengths with baryon number two) and we expect that it will be stable even quantum mechanically (although in the second case there may be some other bound configuration for the deuteron).

As there is no topological reason for the stability of our strings, even less so than for usual cosmic strings which do enjoy and utilise the topology in the transverse direction for part of their stability, an infinite string will reduce its energy per unit length (to zero) by diluting itself (infinitely) along its length. This is not the relevant consideration. We expect our configurations to be important when considering the low energy excitations of lumps with large, but finite baryon number, $N$. The topology of $R^{2}$ indicates that the minimum energy configuration will be a localised, lump of energy, in a field configuration with most likely some large symmetry group. The excitation of this system will always preserve the baryon number. Hence if a string configuration is excited, the relevant energy consideration must be done at fixed baryon number. This boils down to considering the energy per baryon, as we have done above. Excitation of this matter will involve the string configurations, be they only meta-stable. This should correspond to a different low energy phase for the matter described by this energy functional. 
We thank R. Mackenzie and P. Winternitz for useful discussions, and also W.C. Chen and

L. Gagnon for help with the numerical work. This work supported in part by NSERC of Canada and FCAR of Québec. 


\section{References}

1. T.H.R. Skyrme, Proc. Roy. Soc. Lon. $\underline{260}, 127$ (1961)

2. F. Wilczek, A. Zee, Phys. Rev. Lett. 51, 2250 (1983)

Y.S. Wu, A. Zee, Phys. Lett. 147B, 325 (1984)

A.M. Din, W.J. Zakrzewski, Phys. Lett. 146B, 341 (1984)

3. B.M.A.G. Piette, H.J.W. Mueller-Kirsten, D.H. Tchrakian, W.J. Zakrzewski, Phys. Lett. B320, 294 (1994)

B.M.A.G. Piette, B.J. Schroers, W.J. Zakrzewski, Z. Phys. C65, 165 (1995)

4. A.M. Grundland, P. Winternitz, W.J. Zakrzewski, CRM preprint, hep-th/9506068

5. B.M.A.G. Piette, B.J. Schroers, W.J. Zakrzewski, Nucl. Phys. B439 205 (1995) see also A.M. Din, W.J. Zakrzewski, Nucl. Phys. B259, 667 (1985) and references therein.

6. B.J. Schroers, Z. Phys. C61, 479 (1994)

T. Gisiger, M.B. Paranjape, Phys. Rev. D50, 1010 (1994)

R.A. Leese, N.S. Manton, B.J. Schroers, Nucl. Phys. B442, 228 (1995)

T. Gisiger, M.B. Paranjape, Phys. Rev. D51, 3034 (1995)

7. D.H. Lee, C. Kane, Phys. Rev. Lett. 64, 1313 (1990)

S.L. Sondhi, A. Karlhede, S.A. Kivelson, Phys. Rev. B47, 16419 (1993)

K. Moon, H. Mori, K.Y. Yang, S.M. Girvin, A.H. MacDonald, Phys. Rev. B51, 5138 (1995)

C. Nayak, F. Wilczek, IAS preprint, cond-mat/9505081

8. A.A. Belavin, A.M. Polyakov, A.B. Zamolodchikov, Nucl.Phys. B241, 333 (1984)

9. N.S. Manton, Commun. Math. Phys. 111, 469 (1987) 
10. E. Mottola, A. Wipf, Phys. Rev. D39, 588 (1989)

11. E.B. Bogomolnyi, Sov. J. Nucl. Phys. 24, 449 (1976) 


\section{FIGURES}

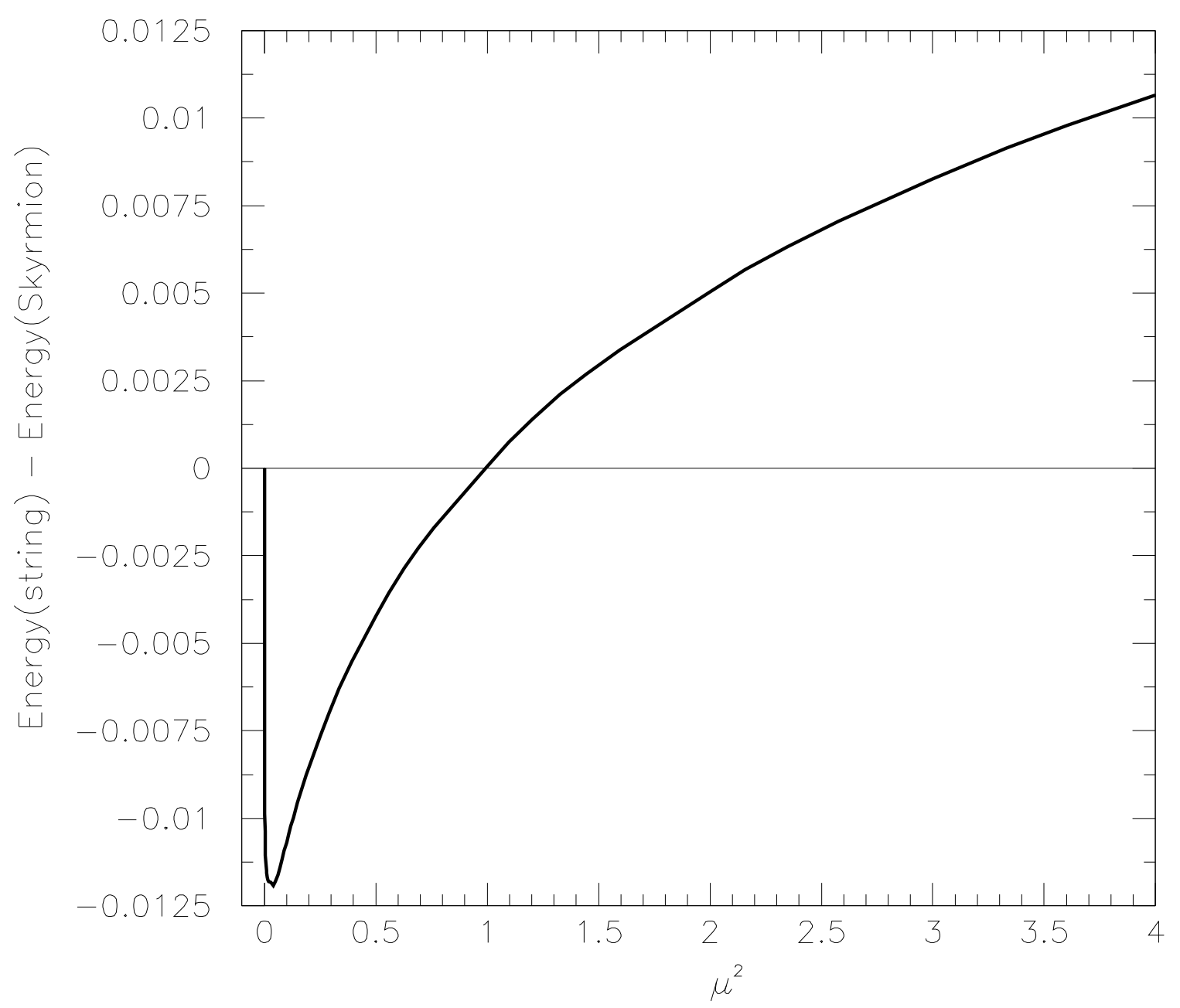

Fig. 1. $E_{\text {string }}-E_{\text {Skyrmion }}$ as a function of $\mu^{2}$. 